\title{
Aplicación de la genética de poblaciones en el ámbito de la medicina
}

\author{
Pablo Octavio-Aguilar, Josefina Ramos-Frías \\ Laboratorio de Genética Evolutiva y Ambiental, Centro de Investigaciones Biológicas, \\ Universidad Autónoma del Estado de Hidalgo, Hidalgo, México
}

\begin{abstract}
Las poblaciones humanas obedecen a los mismos supuestos evolutivos que el resto de los organismos, aunque mezclados con elementos sociales y culturales que pueden promover la expresión de ciertas enfermedades en grupos étnicos específicos, causadas principalmente por la frecuente endogamia. En este trabajo se analiza el principio de Hardy-Weinberg desde un enfoque médico, social y biológico, para entender los procesos evolutivos que dan lugar a las enfermedades autosómicas recesivas. A manera de conclusión se puede señalar que la incidencia de estas enfermedades está inversamente relacionada con los niveles de la variabilidad genética en las poblaciones, variabilidad que depende de eventos de colonización, recolonización y migración, así como de convenciones sociales como el racismo, la estratificación social y la segregación.
\end{abstract}

Palabras clave: ecología humana, enfermedades genéticas congénitas, genética médica, evolución biológica, evolución cultural, flujo genético.

doi: http://dx.doi.org/10.7705/biomedica.v34i2.1540

\section{Application of population genetics in the field of medicine}

Human populations follow the same evolutionary principles as other organisms, although mixed with social and cultural elements, which can result in a high prevalence of certain diseases within specific ethnic groups.

In this work, the Hardy-Weinberg principle is analyzed from a medical, social and biological viewpoint to understand the evolutionary processes of autosomal recessive diseases. It can be concluded that the incidence of these diseases is inversely related to the levels of genetic variability within populations, which depends on colonization, recolonization and migration events, as well as on social conventions such as racism, social stratification and segregation.

Key words: Human ecology; genetic diseases, inborn; genetics, medical; biological evolution, cultural evolution, genetic drift.

doi: http://dx.doi.org/10.7705/biomedica.v34i2.1540

La genética es una rama de las ciencias biológicas que en los últimos años ha tenido un avance rápido, continuo y contundente en diferentes ámbitos relacionados con la biología molecular, la proteómica y la genómica $(1,2)$. Su aportación es fundamental en la educación médica y tiene aplicaciones importantes en la medicina clínica, la salud pública y la investigación aplicada (2). El reconocimiento de la

\footnotetext{
Correspondencia:

Pablo Octavio-Aguilar, Laboratorio de Genética Evolutiva y Ambiental, Centro de Investigaciones Biológicas, Universidad Autónoma del Estado de Hidalgo, Carretera Pachuca-Tulancingo km 4.5, Ciudad del Conocimiento, Colonia Carboneras, Mineral de la Reforma, Hidalgo, México, C.P. 42184

Teléfono: (52 771) 7172 0000, extensión 6649 aguilpo@yahoo.com.mx
}

Recibido: 11/01/13; aceptado: 07/12/13 importancia de la genética ha generado un verdadero problema para ubicarla en los currículos de la carrera de medicina, un problema que solo se ha resuelto de forma parcial en la mayoría de las facultades, aun cuando desde 1962 se ha puesto de manifiesto la necesidad de desarrollar cursos especializados que den cuenta de los avances en la materia (3).

La genética de poblaciones es solo una parte de la genética y se encarga del estudio comparativo de la variación entre individuos. Su enfoque constituye una visión matemática de la distribución, así como de la dinámica de los alelos y genotipos dentro de las poblaciones y entre ellas (4). Entendida de esta forma, la mayoría de los médicos ignora sus alcances y llega a pensar que no tiene relación con su campo.

\section{Contribución de los autores:}

Pablo Octavio Aguilar: escritura del manuscrito

Josefina Ramos-Frías: compilación de la información, revisión y corrección del manuscrito 
En este ensayo se pretende dar una idea, útil para cualquier estudiante o médico, de las aplicaciones que la genética de poblaciones podría aportar a su formación. Esta información también es relevante para entender la incidencia de algunas enfermedades hereditarias en grupos étnicos específicos.

\section{Variación}

El hecho de que dos individuos sean diferentes implica que cada uno puede tener herramientas distintas para afrontar el mismo problema adaptativo, y es por ello que la variabilidad genética representa el potencial evolutivo de las especies (5). Lo más importante es saber de dónde viene esa variación y cuáles son los factores que podrían aumentar o disminuir tales diferencias.

La variación puede establecerse con base en las diferencias de los nucleótidos adenina, timina, guanina y citosina; en una secuencia de ADN, cada base distinta constituye un alelo y, cada posición específica, un locus. A un nivel superior, una secuencia funcional de nucleótidos (codificadora o que se puede modificar después de la replicación) se considera un alelo, siempre que existan variantes de los productos de la expresión entre individuos; la posición de dicha secuencia dentro del genoma sería un locus. Si un individuo hereda un alelo de su padre y otro diferente de su madre, se puede decir que es heterocigótico; si ambos padres le heredan el mismo alelo, entonces sería homocigótico. De esta forma, resulta fácil comprender cuán diferentes somos considerando la proporción de alelos idénticos o diferentes. En las poblaciones también hay medidas similares, como son el número efectivo de alelos por locus, el porcentaje de locipolimorfos y los promedios de la heterocigosis observada y la esperada (6).

La manera en que los alelos y los genotipos se distribuyen dentro de las poblaciones y entre ellas se conoce como estructura genética y da lugar a las medidas de diferenciación y de endogamia conocidas como el coeficiente de consanguinidad (F) de Wright (6-8).

Toda esta información solo nos dice si las poblaciones y los individuos son distintos genéticamente, pero no esclarece las causas de dichas diferencias. Para ello necesitamos entender varios factores y procesos, entre ellos los biológicos, los sociales y los económicos.

\section{Causas y consecuencias}

Todos los seres humanos tenemos alrededor de 32.180 genes ordenados en 46 cromosomas, lo que equivale a unos 2.900 millones de pares de bases; hasta el 2005 se conocía la identidad de menos de la mitad de estos genes (9). En la actualidad, se puede asegurar que $99,9 \%$ de la información contenida en los cromosomas es idéntica para todo el género humano y que, aproximadamente, $5 \%$ se ha conservado sin alteraciones en los últimos 200 millones de años. Además, se puede asegurar que las secuencias funcionales (genes codificadores, regiones reguladoras y secuencias que pueden sufrir metilación) solo constituyen el $2 \%$ del genoma completo (10). Entonces, ¿qué nos hace tan diferentes? A este respecto se puede decir con seguridad que las pequeñas diferencias entre individuos se encuentran en loci funcionales y que cada población humana tiene diferentes frecuencias alélicas para tales loci.

Las causas de estas diferencias tienen que ver con fuerzas evolutivas, como la selección natural, la endogamia, las mutaciones, la deriva genética y el flujo génico (8), así como con cuestiones meramente sociales, como la preferencia sexual, el racismo, el aislamiento geográfico y la migración histórica $(11,12)$. Como resultado de estos procesos, hoy se encuentran diferencias importantes en la frecuencia de ciertos alelos en las poblaciones humanas (cuadro 1).

La mayoría de estas diferencias se debe a procesos de migración y aislamiento (13) o a la eliminación de la variación por el efecto fundador (14) y a los llamados 'cuellos de botella' (17), situaciones que pueden fluctuar a lo largo de la historia de las poblaciones, aunque no acarrean ningún peligro para los portadores o sus familias. Es en el contexto de ciertas enfermedades genéticas que las diferencias cobran importancia. Algunos grupos raciales tienen una mayor propensión a

Cuadro 1. Ejemplos de alelos con diferente frecuencia en poblaciones humanas

Sistema sanguíneo ABO

Actividad de la lactasa

Alcohol deshidrogenasa
El alelo B es común entre los asiáticos y no existe en los nativos americanos (14).

Alelo de baja eficacia más frecuente en asiáticos y africanos (80 a $95 \%$ ), menos común en europeos y caucásicos (17 a $48 \%$ ) (15).

El alelo $\mathrm{ADH}_{2}$ es más común en japoneses (90\%) que entre europeos (15\%) (16). 
Cuadro 2. Trastornos genéticos con incidencia diferencial dentro de algunos grupos étnicos

\begin{tabular}{|c|c|}
\hline Fibrosis quística & $\begin{array}{l}\text { Elevada frecuencia en poblaciones caucásicas (1/40 a 1/50), baja en poblaciones de Asia } \\
\text { y África (18). }\end{array}$ \\
\hline Anemia falciforme & Se presenta en 1/20 afroamericanos en comparación con 1/200 hispanoamericanos (19). \\
\hline Fenilcetonuria & $\begin{array}{l}\text { Elevada frecuencia en europeos de origen celta (1/67 a 1/90), baja en japoneses y } \\
\text { finlandeses }(1 / 500)(20) \text {. }\end{array}$ \\
\hline Enfermedad de Tay-Sachs & Elevada frecuencia en judíos asquenazíes (1/60), cientos de veces menor en otros grupos (21). \\
\hline Distrofia miotónica & $\begin{array}{l}\text { Se presenta en } 1 / 50.000 \text { personas en Europa, prácticamente no existe en el África } \\
\text { subsahariana y } 1 / 950 \text { personas la padecen en algunas regiones de Quebec (22). }\end{array}$ \\
\hline Hipercolesterolemia familiar & $\begin{array}{l}\text { Elevada frecuencia en ciertas regiones de Quebec }(1 / 244) \text { y entre afrikáneres }(1 / 140) \text {. } \\
\text { Para el resto de los grupos la frecuencia es de } 1 / 1.000(23) \text {. }\end{array}$ \\
\hline
\end{tabular}

padecer trastornos hereditarios que deben tenerse en cuenta en el momento de hacer la historia clínica (cuadro 2).

\section{Violación de los supuestos}

De acuerdo con el principio de Hardy-Weinberg, las frecuencias alélicas en una población tienden a ser estables en el tiempo, generación tras generación, siempre y cuando se cumplan los siguientes cinco supuestos: que todos los individuos tengan la misma probabilidad de sobrevivir; que no haya mutaciones; que los apareamientos sean aleatorios; que las poblaciones sean grandes; que todos los alelos estén bien representados, y que no haya entrada ni salida de alelos.

La violación de cualquiera de estos supuestos conlleva la desviación del equilibrio y modifica la frecuencia de los alelos y de los genotipos dentro de las poblaciones. Así, el modelo del equilibrio se convierte en el modelo nulo de una población ideal que permite la comparación con las poblaciones reales.

Las especies pueden o no estar en equilibrio dependiendo de la dinámica de sus poblaciones en un momento dado, y los seres humanos no somos la excepción.

En muchas culturas, los humanos delimitan su proceder evolutivo más por reglas sociales y costumbres que por comportamientos naturales comunes al resto de las especies. Los seres humanos seleccionamos a nuestras parejas por diversas razones biológicas, sociales, económicas y culturales, atraídos en muchas ocasiones por elementos subjetivos que escapan a nuestro entendimiento, pero que tratamos de racionalizar, y que, en esencia, podrían responder a mecanismos bioquímicos (24). Tendemos a escoger cierto patrón fenotípico estereotipado que nos aleja de la panmixia (aleatoriedad reproductiva), aunque debe anotarse que esta tendencia podría ser producto del incremento reciente en las migraciones, lo que ha permitido la comparación y el intercambio de patrones de belleza antes bien definidos en el interior de los grupos étnicos (25). Además, en ciertas comunidades los emparejamientos se dan dentro de las familias con la excusa de conservar la pureza de la raza $(26,27)$; por otra parte, los migrantes diseminan sus genes por fuera de su grupo y, en general, mutamos al exponernos a los agentes químicos y físicos que usamos en las industrias, fundamos pueblos grandes y chicos, nos aislamos, eliminamos individuos, hacemos guerras religiosas, etc. Hay, así pues, un sinfín de comportamientos y tendencias que violan los principios mencionados y que nos permiten elucubrar sobre el por qué algunas enfermedades son más frecuentes en ciertos grupos que en otros, fenómeno que no es exclusivo de los seres humanos, ya que, de hecho, las violaciones a los supuestos de Hardy-Weinberg son el común denominador en la mayoría de las especies animales y vegetales.

Se podría pensar que el uso de esta teoría es inocuo, dado que nunca se cumple, pero estos supuestos deben abordarse desde una visión popperiana, en la que su negación nos confirma la existencia de fuerzas evolutivas y nos ayuda a definir cuáles son las más importantes cuando actúan en conjunto.

\section{Primer supuesto: cuando no todos sobreviven}

El proceso de la selección natural se puede resumir de la siguiente forma:

1) las poblaciones pueden incrementar su densidad en proporción geométrica siempre y cuando cuenten con recursos ilimitados;

2) como los recursos son limitados por el ambiente, podemos decir que este impone una presión selectiva que origina una lucha por la 
existencia. Debe anotarse que cada sitio impone diferentes competencias que pueden suceder simultáneamente, generando procesos evolutivos paralelos divergentes o convergentes;

3) las personas muestran variabilidad fenotípica en aquellos caracteres que son relevantes para la competencia, como son una mayor resistencia a un agente patógeno o mejores rasgos sexuales para atraer potenciales parejas;

4) la mortalidad y la reproducción están diferenciadas con respecto a tales caracteres, y

5) como, al menos, parte de la variabilidad fenotípica es heredable, el cambio evolutivo resulta cuando los sobrevivientes de esta lucha procrean, es decir, cuando hay descendencia con modificación $(28,29)$.

En los seres humanos existe un caso muy interesante en el que la selección natural ha creado las condiciones para que se dé una mayor incidencia de cierta enfermedad genética en algunas regiones de África porque confiere una resistencia significativa a un factor de selección local: la malaria.

La anemia falciforme es una enfermedad hereditaria humana caracterizada por eritrocitos escasos, alargados, delgados y con forma de media luna, además de un número extraordinariamente elevado de células inmaduras. Cuando un individuo ha heredado de ambos progenitores (homocigótico) el alelo para esta enfermedad, su esperanza de vida es de apenas unos años. Sin embargo, si los individuos reciben solo un alelo enfermo (heterocigóticos), sufren una enfermedad más leve llamada rasgo de la anemia falciforme, en la cual solo $1 \%$ de los eritrocitos son falciformes, y pueden llevar una vida normal si evitan la fatiga. La investigación sobre la persistencia de este alelo defectuoso en África llevó al descubrimiento de que en individuos heterocigóticos aporta una pequeña aunque significativa resistencia a formas letales de la malaria, cuya incidencia es elevada en las mismas regiones de ese continente. De esta forma, la selección natural ha generado la persistencia del alelo en zonas donde confiere ventajas ante una presión selectiva mucho mayor (30).

\section{Segundo supuesto: los mutantes}

Una mutación es la alteración en la secuencia de ADN de una célula, lo que abarca desde el cambio, pérdida o inserción de un solo par de bases nitrogenadas hasta la ganancia o pérdida de cromosomas enteros o de parte de ellos. Dichas alteraciones pueden ser espontáneas o causadas por agentes químicos, por radiación o por errores durante los procesos de replicación y reparación del ADN (31). En la actualidad, se conocen alrededor de 4.000 trastornos mutagénicos; la mayoría raramente ocurre y afecta a un solo individuo entre miles o millones de personas. La fibrosis quística es uno de los trastornos más comunes; alrededor de $5 \%$ de la población de los Estados Unidos lleva, al menos, una copia del gen defectuoso.

Para que una mutación pueda fijarse en una población, debe mejorar las expectativas de supervivencia o reproducción del portador y debe poder transferirse a su descendencia, lo que raramente sucede. La mayoría de las mutaciones ocurren con una frecuencia muy baja, aproximadamente entre 0,97 y $1,8 \times 10^{-8}$ nucleótidos por generación, ya que existen regiones en el genoma que tienen tasas de mutación más elevadas que otras $(32,33)$.

Además, las mutaciones por lo general disminuyen la adecuación de los portadores y la mayoría son de tipo recesivo, es decir que para manifestarse físicamente, los alelos de ambos progenitores deben presentar la mutación (34). Por otro lado, la mayoría de las células de un organismo son somáticas, por lo que los efectos de las mutaciones comunes y espontáneas desaparecen en la siguiente generación, aunque pueden ser devastadores para el individuo, como sucede con el cáncer. Es importante mencionar que la sensibilidad frente a las mutaciones espontáneas en células no germinativas (somáticas) sí puede tener un componente hereditario que debe tomarse en cuenta durante el diagnóstico, tal como la predisposición genética al retinoblastoma, al cáncer colorrectal y a los neuroblastomas, entre otras enfermedades $(35,36)$.

Existen múltiples ejemplos de enfermedades producidas por mutaciones en los seres humanos, todas las cuales se manifiestan en individuos homocigóticos recesivos. Entre estas se cuentan la poliquistosis renal infantil, la fibrosis quística, la distrofia miotónica, el albinismo, la displasia espóndilo-torácica, la polidactilia, la gangliosidosis, la histiocitosis recesiva, la acondroplasia, la acidosis láctica, el enanismo cifomélico y la sindactilia, para mencionar al menos una docena, todas ellas causadas por alelos mutantes con menor adecuación.

Se puede afirmar que $98,6 \%$ de las mutaciones son deletéreas, ya sea porque simplemente no se transcriben al ocurrir en secuencias de ADN 
silentes, o porque sus portadores mueren irremediablemente antes de dejar descendencia (37). Sin embargo, las mutaciones no desaparecen de las poblaciones, ya que tarde o temprano vuelve a aparecer una secuencia modificada.

\section{Tercer supuesto: la selección sexual}

Hay, al menos, tres violaciones al supuesto de los apareamientos aleatorios en los seres humanos: la estratificación, el emparejamiento dirigido y la consanguinidad y, si bien podrían encontrarse ejemplos en otras especies animales y vegetales, en el caso de los seres humanos merece la pena mencionar estos componentes sociales y culturales.

La estratificación consiste en la formación de subgrupos dentro de las poblaciones, ya sea por edad, por cultura o por procedencia. El mejor ejemplo es la sociedad estadounidense, en la cual caucásicos, afroamericanos, nativos americanos, hispanos y asiáticos conviven abiertamente pero se segregan entre sí, de forma que los emparejamientos se dan dentro de cada grupo, manteniéndose así las diferencias genéticas durante varias generaciones. Se podría suponer que en Estados Unidos existe una gran diversidad genética, pues se trata de una sociedad incluyente, pero estaríamos equivocados, ya que la diversidad no se distribuye de manera homogénea sino que los subgrupos tienden a mantenerse por cuestiones meramente sociales, económicas y culturales, lo cual genera una estructura genética bien definida (38). Cabe aclarar que la distribución de la variación genética dentro de las poblaciones y entre ellas responde a un patrón de flujo histórico con intercambios esporádicos, lo que diferencia la estratificación de los emparejamientos dirigidos. Para entender esto basta con observar que hay una mayor diferenciación genética entre poblaciones geográficamente cercanas pero que se han caracterizado por segregarse mediante guerras o la eliminación de poblados completos, como es el caso de los yanomamis (39); por otra parte, en todo el planeta las personas con acondroplasia congénita conforman un grupo que sí se diferencia de quienes no la padecen, independientemente de su localización geográfica (40). El primero sería un ejemplo de estratificación y, el segundo, de emparejamiento dirigido.

Para profundizar más en estas diferencias, se debe anotar que el emparejamiento dirigido ocurre si la selección de parejas se da dentro de grupos con un acervo genético común, ya sea por cultura, aprobación social o, incluso, por apariencia física. En Estados Unidos, por ejemplo, se lleva a cabo una convención anual de "gente pequeña", en la cual las personas con síndrome acondroplásico congénito acuden a conferencias médicas y actividades orientadas a dar respuesta a sus necesidades especiales, y es precisamente en estos eventos en los que se forman parejas, ya que los asistentes tienen más probabilidades de conocer a alguien que comparte el mismo fenotipo. Esto parecería discriminatorio, pero responde a la necesidad de incrementar la probabilidad real de satisfacer el deseo de procreación de un grupo dado. De alguna manera, tal comportamiento obedece a miedos fundados en el rechazo a su condición, el cual también parece tener una trasfondo evolutivo (41), pues saben claramente que habría una mayor probabilidad de tener hijos con el mismo padecimiento. Así, pues, se tienen los dos extremos: el rechazo total al emparejamiento con personas que padecen el mismo síndrome o el emparejamiento preferencial con personas similares por temor al rechazo (42).

Por último, la consanguinidad también se da cuando los matrimonios se realizan no solo en el interior de un grupo genético común, sino dentro una misma familia. Este sería el máximo nivel de endogamia posible al contemplar el sesgo sexual de la panmixia. Existen muchas razones que explican la consanguinidad, casi todas ideológicas o de mantenimiento del poder o la riqueza. Como un ejemplo de las consecuencias de la consanguinidad se puede mencionar la hemofilia, afección caracterizada por la ineficacia de los factores de coagulación que han sufrido mutaciones. Estos alelos están asociados al cromosoma X, por lo que se manifiestan en el sexo masculino. Sin embargo, cuando un enfermo se reproduce con una familiar portadora del mismo alelo hay $25 \%$ de probabilidad de tener una hija enferma y la mitad de la descendencia será portadora. Esto ya ha sucedido con la descendencia de la reina Victoria, cuyo linaje se caracterizó por el arreglo de matrimonios consanguíneos en todas las cortes europeas.

Como dato curioso vale la pena anotar que en pocos casos existe la tendencia a escoger una pareja lo más alejada posible del fenotipo parental; algunos autores creen que esta sería una tendencia evolutiva inconsciente para aumentar la variabilidad genética relacionada con la presencia de diferentes quimiotipos del complejo mayor de histocompatibilidad humana (43). Esto constituye otro ejemplo de emparejamiento dirigido pero a la inversa, lo que se podría llamar exogamia preferencial (44). 


\section{Cuarto supuesto: el azar}

El hecho de escoger pareja siempre tiene un cierto grado de aleatoriedad. Nos guiamos por el fenotipo sin saber a ciencia cierta la información incluida, a manera de sorpresa, en el paquete completo. Muchas veces es demasiado tarde cuando las mujeres se enteran de que su marido tiene tendencia a la calvicie o a la obesidad, y la posibilidad de que sus vástagos hereden dichas tendencias les crea verdadera ansiedad.

En poblaciones de gran tamaño, la probabilidad de encontrar genes 'defectuosos' debería ser muy baja, puesto que su frecuencia inicial no podría mantenerse si tales defectos disminuyen la adecuación de los portadores. Pero cuando las poblaciones son pequeñas, muchas veces no hay más remedio que resignarse a aceptar la pareja que nos tocó con todos sus defectos; de todas maneras, siempre hay cierta disminución en la fecundidad de tales poblaciones, así como una tendencia a elegir la misma pareja, por lo que se produce algo de promiscuidad (27). Las poblaciones pequeñas son frecuentes en sitios inaccesibles y aislados o en centros de población fundados recientemente o cuando la población se ha reducido (cuellos de botella) por efecto de la migración o las enfermedades (17). Este fenómeno no es exclusivo de los seres humanos, pues es la principal causa de la pérdida de variabilidad genética en especies como el guepardo y la vaquita marina.

Por otro lado, la homogeneidad genética de una población, que se produce porque los individuos provienen de unos pocos pobladores originales, se conoce como efecto fundador; un indicador de tal efecto es la repetición de los apellidos en ciertas localidades, aunque esta característica solo permite seguir los linajes paternos. Si a esta situación se le suma el efecto del aislamiento geográfico, nos encontramos con verdaderas islas donde a los pobladores no les queda más remedio que casarse con sus parientes, lo cual genera una depresión por endogamia. Es en estos casos cuando el azar juega en contra al producir mayor cantidad de individuos homocigóticos simplemente porque no hay más alelos de dónde escoger, tal como ha sucedido en las poblaciones donde la colonización histórica respondió al aislamiento genético y geográfico de las comunidades y a corrientes de colonización que poco a poco fueron contrarrestando el efecto de la endogamia $(45,46)$.

Finlandia, situada en el borde del mundo habitable, es una de las islas genéticas mejor estudiadas. Se conocen, al menos, 35 enfermedades hereditarias con una incidencia anormal en estas gélidas regiones. Varios estudios moleculares han demostrado que la población total de esta gran isla proviene de dos grupos de migrantes llegados hace 4.000 años desde el este de Europa y hace 2.000 años desde regiones meridionales del continente por vía marítima. Esto generó dos grupos de población que se fueron mezclando gradualmente en corrientes intermitentes hasta producir un acervo genético común, más relacionado con el segundo proceso de migración. Este es un claro ejemplo del efecto fundador seguido de un proceso de endogamia a partir de una selección aleatoria de genes iniciales, fenómeno conocido como deriva genética (47). Como consecuencia, las poblaciones más relacionadas con el primer efecto fundador, llegadas desde el norte, presentan una alta incidencia de coroideremia, enfermedad que se caracteriza por la pérdida progresiva de la visión, cuyo primer síntoma suele ser la incapacidad para la visión nocturna, la cual puede aparecer en la infancia temprana. La coroideremia es una enfermedad degenerativa recesiva de la retina, ligada al cromosoma $X$ del gen que codifica la proteína Rab escolta 1, que afecta solamente a los varones. Por el contrario, las poblaciones fundadas en el segundo periodo de colonización, provenientes de Europa meridional por vía marítima, presentan una mayor incidencia de hiperornitinemia, caracterizada por niveles excesivos de ornitina en la sangre causados por una deficiencia de la enzima mitocondrial ornitina aminotransferasa. En general, sus síntomas son muy similares a los de la coroideremia, pero dado que no está relacionada con el cromosoma sexual, no es exclusiva del sexo masculino.

Se puede afirmar, entonces, que los pocos individuos que dieron origen a la población de Finlandia tenían, por azar, algunos defectos genéticos que se fijaron en la comunidad porque había una mayor probabilidad de encontrar individuos portadores.

\section{Quinto supuesto: la migración}

El flujo de alelos entre poblaciones tiene el efecto inmediato de la aparición de nuevos caracteres en las poblaciones receptoras. Si este flujo génico es continuo, se tiende a la homogeneidad e igualdad de las poblaciones implicadas. Si el flujo se interrumpe, entonces aparecen nuevas diferencias.

Muchas veces estas corrientes migratorias ocurren por oleadas, manteniendo una dinámica intermitente de rasgos que aparecen y desaparecen en las poblaciones o que se fijan una vez que se suspenden los procesos migratorios. Gracias a 
las nuevas tecnologías basadas en la variación de los marcadores mitocondriales y los asociados al cromosoma y, así como a diversos vestigios fósiles, se han podido reconstruir las corrientes migratorias que han dado origen a las poblaciones humanas (48-50).

La humanidad inició su historia de migración hace, aproximadamente, 200.000 años en el valle del Rift, en la región subsahariana oriental de África. Los primeros seres humanos eran pocos, compartían un acervo genético común y eran nómadas que se alimentaban de los recursos naturales de cada región donde se asentaban. En cada sitio gradualmente desplazaban a los homínidos con quienes inicialmente convivían.

El primer intento por dejar el continente africano tuvo lugar hace unos 110.000 años, evento del cual solo se tienen registros fósiles en el Medio Oriente (13). Una segunda corriente migratoria llegó a la región del Indo rodeando la península arábiga hace unos 70.000 años. Para ese momento se tiene registro de tres haplotipos (variedades) mitocondriales que nos hablan de sendos linajes distintos, es decir, tres grupos diferentes cuyo origen fue la misma región africana (51). Posteriormente, hace unos 50.000 años, una tercera corriente salió de África hacia las llanuras fértiles de Mesopotamia y el Medio Oriente, donde nació la agricultura. A partir de ese momento, y hasta hace unos 30.000 años, los humanos se extendieron por Europa y Asia, colonizando nuevos ambientes y generando cuellos de botella con cada nuevo asentamiento, lo que permitió la creciente diferenciación de las poblaciones conforme se iban alejando del centro de radiación y fijando mutaciones adaptativas locales (52). Así surgieron las primeras civilizaciones en la región del Mediterráneo y del Medio Oriente, seguidas de los centros de población en China e India. A partir de esas primeras corrientes (hace 70.000 años), muchas poblaciones descendieron hacia Indonesia y alcanzaron Australia hace unos 60.000 años (53).

Por último, hace unos 25.000 años, un puñado de hombres llegó a América proveniente de Asia; esos pequeños grupos llevaban consigo una información totalmente diferente, que terminó separándolos del resto de la humanidad, estableciendo diferencias que permanecieron como huellas en sus genes y que hoy nos permiten reconstruir el árbol filogenético general de lo que sucedió. Cabe mencionar que algunos grupos se aislaron desde sus inicios, como los pobladores de Papúa y los kabish de las
Ilanuras de Mongolia, y por ello constituyen grupos más aislados genéticamente que el resto de las comunidades estudiadas (13).

Como ya se ha mencionado, dicho aislamiento conlleva una homogeneidad genética que hace a una población más proclive a adquirir enfermedades, las cuales se extienden rápidamente entre los miembros de tales sociedades aisladas. Si bien estos padecimientos no son hereditarios, la falta de resistencia sí lo es. Como ejemplo de ello se pueden citar; la propensión a adquirir la esquistosomiasis, ligada a la región q31-q33 del cromosoma 5, y que está presente sobre todo en poblaciones de Brasil y Sudán $(54,55)$; la predisposición a la tuberculosis, ligada a la región 2 q35 y conocida hoy como TBS1 (56), la cual se identificó a partir de una epidemia entre aborígenes canadienses; y la predisposición a padecer lepra de grupos étnicos vietnamitas e indios asociada con la región $6 q 25$ y la 10p13, respectivamente $(57,58)$.

\section{Conclusiones}

Se puede afirmar que la incidencia de las enfermedades autosómicas recesivas en los seres humanos depende de la disminución de la variabilidad genética, representada por la pérdida de alelos, de genotipos, de polimorfismo, etc. Esta disminución puede ocurrir de manera gradual debido a diversos eventos de colonización, recolonización y migración, o, en muchos casos, verse fomentada por las convenciones sociales que favorecen la endogamia, como son el racismo, los matrimonios consanguíneos, y la estratificación económica de los grupos sociales, y por disminuciones abruptas en la diversidad debidas al efecto fundador y a los llamados cuellos de botella. Por último, la fijación de estas pérdidas genera diferencias significativas entre las poblaciones, lo que se ve favorecido por el aislamiento geográfico y la ineficacia en el flujo génico.

Todas estas excepciones del equilibrio de HardyWeinberg han quedado registradas en la memoria genética de las poblaciones y gracias a ello ha sido posible reconstruir la historia evolutiva del hombre. Sin embargo, como entidades biológicas que somos, los seres humanos respondemos a los mismos principios generales que rigen al resto de los seres vivos $y$, salvo algunas particularidades sociales, no nos comportamos de manera tan diferente a una planta o a un animal.

\section{Agradecimientos}

A Crystian Venegas Barrera, por las observaciones al manuscrito, al posgrado en Biología del 
Instituto Tecnológico de Ciudad Victoria, por la invitación a participar en su foro interno de investigación con la disertación que originó este documento.

\section{Conflicto de intereses}

Al ser el presente estudio parte del trabajo académico de los autores, no existe conflicto de intereses alguno con las instituciones. Los trabajos referidos han sido debidamente citados.

\section{Financiación}

Este documento se realizó en las instalaciones del Instituto Tecnológico de Ciudad Victoria, Tamaulipas, México, con financiación de la Secretaría de Educación Pública y la Dirección General de Educación Superior Tecnológica de México, y fue corregido en el Instituto de Ciencias Biológicas de la Universidad Autónoma del Estado de Hidalgo, México.

\section{Referencias}

1. Ruffin A. Report VI: Contemporary issues in medicine: Genetics education. Washington, D.C.: Association of American Medical Colleges; 2004.

2. Suárez OF, Zárate MI. Introducción y justificación para la creación del Grupo de Genética Médica de la Asociación Colombiana de Genética Humana. Junta Directiva de la ACGH en su reunión del día 4 de septiembre de 2003. Acta Nº37 de 2003. Bogotá, D.C.: Asociación Colombiana de Genética Humana; 2003.

3. Book J, Ceppellini R, Clarke FF, Frota-Pessoa O, Klein $\mathbf{D}$, Lamy $\mathbf{M}$, et al. La enseñanza de la genética en las facultades de medicina y en cursos de perfeccionamiento. Primer Informe del Comité de Expertos en Genética Humana. Serie de Informes Técnicos No. 238. Ginebra: Organización Mundial de la Salud; 1962.

4. Hartl D, Clark A. Principles of population genetics. Third edition. Sunderland, MA: Sinauer Associates Press; 1997.

5. Lande R. Extinction risk from anthropogenic, ecological and genetic factors. In: Landweber LF, Dobson AP, editors. Genetic and the extinction of species. Princeton, NJ: Princeton University Press; 1999.

6. Wright $\mathbf{S}$. The genetical structure of populations. Ann Eugen. 1951;15:323-54.

7. Wright S. Evolution and the genetics of populations. Vol. 2. The theory of gene frequencies. Chicago, London: University of Chicago Press; 1969.

8. Wright S. Evolution and the genetics of populations. Vol. 4: Variability within and among natural populations. Chicago, London: University of Chicago Press; 1978.

9. Cervantes-García D, González RC, Mayek N. Proyecto genoma humano: situación actual y perspectivas. Investigación y Ciencia. 2005;13:56-63.

10. ENCODE Project Consortium, Birney E, Stamatoyannopoulos JA, Dutta A, Guigó R, Gingeras TR, et al. The ENCODE Project Consortium. Identification and analysis of functional elements in $1 \%$ of the genome by the ENCODE pilot project. Nature. 2007;447:799-816. http://dx.doi.org/10. 1038/nature05874

11. Jablonski NG, Chaplin G. The evolution of human skin coloration. J Hum Evol. 2000;39:57-106. http://dx.doi.org/10. 1006/jhev.2000.0403

12. Risch N, Burchard E, Ziv E, Tang H. Categorization of humans in biomedical research: Genes, race and disease. Genome Biol. 2002;3:1-12. http://dx.doi.org/10.1186/gb2002-3-7-comment2007

13. Race RR, Sanger R. Blood groups in man. Second edition. New York: Blackwell Publications; 1975.

14. Zúñiga GA. Intolerancia a la lactosa. Rev Med Hond. 1995;63:20-3.

15. Escarabajal MD. Alteraciones genéticas relacionadas con el alcoholismo. Rev Neurología. 2003;37:471-80.

16. Rosenberg NA, Pitchard JK, Weber JL, Cann HM, Kidd KK, Zhivotovsky LA, et al. Genetic structure of human populations. Science. 2002;298:2381-5. http://dx.doi.org/10. 1126/science. 1078311

17. Behar DM, Hammer MF, Garrigan D, Villems R, BonneTamir B, Richards M, et al. MtDNA evidence for a genetic bottleneck in the early history of the Ashkenazi Jewish population. Eur J Hum Genet. 2004;12:355-64. http://dx.doi. org/10.1038/sj.ejhg.5201156

18. Congress US Office of Technology Assessment. Cystic fibrosis and DNA tests: Implications of carrier screening, OTA-BA-532. Washington, DC: U.S. Government Printing Office; 1992.

19. Serjeant GR. Sickle-cell disease. New York: Oxford University Press; 1985.

20. Campistol PJ, Cambra FJ, Lambruschini N, Vilaseca MA, Fusté ME, Maollas J, et al. PKU? Barcelona: Editorial Gráficas Signo; 1997.

21. Novoa F, Colombo M, Clericus J. Enfermedad de Tay Sachs. Rev Chil Ped. 1974;45:495-9. http://dx.doi.org/10. 4067/S0370-41061974000600003

22. Sierra MP, Losada J, Rufo A, Lucas M. Estudio genético molecular en la distrofia miotónica congénita. Rev Neurología. 1997;25:833-6.

23. Atienza MG. Hipercolesterolemia familiar: evaluación del diagnóstico genético mediante micromatrices de ADN. Consulta técnicas: CT2006/02. Santiago de Compostela: Consellería de Sanidade, Axencia de Avaliación de Tecnoloxías Sanitarias de Galicia, Avalia-t. Serie Avaliación de tecnoloxías; 2006.

24. Flores-Rosales G. La fórmula química de Cupido. Revista Digital Universitaria (UNAM). 2008;9:1-7.

25. Thornhill R, Gangestad SW. Human facial beauty: Averageness, symmetry and parasite resistance. Hum Nat. 1993;4:237-69. http://dx.doi.org/10.1007/BF02692201

26. Root $\mathbf{M}$. The use of race in medicine as a proxy for genetic differences. Philos Sci. 2003;70:1173-83.

27. Bittles AH. Endogamy, consanguinity and community genetics. J Genet. 2002;81:91-8. http://dx.doi.org/10.1007/ BF02715905

28. Marone L, Milesi F, González R, Mezquida ET, López J, Cueto V. La teoría de la evolución por selección natural 
como premisa de la investigación ecológica. Interciencia 2002;27:137-42.

29. Demetrius L. The demographic evolution of human populations: The role of selection and environmental factors. Demography. 1989;26:353-72. http://dx.doi.org/10.2307/ 2061598

30. National Institutes of Health, National Health, Heart, Lung, and Blood Institute. La anemia falciforme. Publication No. 98-4086. Bethesda, MD: National Institutes of Health; 1998.

31. Friedberg EC, Walker GC, Siede W. DNA repair and mutagenesis. Washington, DC: ASM Press; 1995.

32. Nachman MW, Crowell SL. Estimate of the mutation rate per nucleotide in humans. Genetics. 2000;156:297-304.

33. Scally A, Durbin R. Revising the human mutation rate: Implication for understanding human evolution. Nat Rev Genet. 2012;13:745-53. http://dx.doi.org/10.1038/nrg3295.

34. Nei M. The new mutation theory of phenotypic evolution. Proc Natl Acad Sci USA. 2007;104:12235-42. http://dx.doi. org/10.1073/pnas.0703349104

35. Schwechheimer K, Cavenee WK. Genetics of cancer predisposition and progression. Clin Investig. 1993;71:488 502. http://dx.doi.org/10.1007/BF00180066

36. Kazsuba M, Sikorski A, Odwazna J, Wojciechowicz J. Diagnostic microarray test for a genetic predisposition to hereditary nonpolyposis colorectal cancer (HNPCC). Hered Cancer Clin Pract. 2012;10(Suppl.4):A23. http://dx.doi. org/10.1186/1897-4287-10-S4-A23

37. Kondrashov AS. Measuring spontaneous deleterious mutation process. Genetica. 1998;102-103:183-97. http://dx. doi.org/10.1023/A:1017085731998

38. Cerda-Flores RM, Villalobos-Torres MC, Barrera-Saldaña HA, Cortés-Prieto LM, Barajas LO, Rivas F, et al. Genetic admixture in three Mexican mestizo populations based on D1S80 and HLA-DQA1 Loci. Am J Hum Biol. 2002;14:25763. http://dx.doi.org/10.1002/ajhb.10020

39. Guerreiro JF, Figueiredo MS, Santos SBF, Zago MA. $\beta$-globin gene cluster haplotypes in Yanomama indians from the Amazon region of Brazil. Hum Genet. 1992;89:629-31.

40. Read AP, Donnai D. What can be offered to couples at (possibly) increased genetic risk? J Community Genet. 2012; 3:167-74. http://dx.doi.org/10.1007/s12687-012-0105-1

41. Park JH, Faulkner J, Schaller M. Evolved diseaseavoidance processes and contemporary anti-social behavior: Prejudicial attitudes and avoidance of people with physical disabilities. J Nonverbal Behav. 2003;27:65-87. http://dx.doi. org/10.1023/A:1023910408854

42. Pantano L. Hacia una mayor comprensión en el campo de la discapacidad. Situación social de las personas con acondroplasia. Boletín de Lecturas Sociales y Económicas. 1996;3:33-51.

43. Beauchamp GK, Yamazaki K. HLA and mate selection in humans: Commentary. Am J Hum Genet. 1997;61:494-6. http://dx.doi.org/10.1086/515521

44. Laurent R, Toupance B, Chaix R. Non-random mate choice in humans: Insights from a genome scan. Mol Ecol. 2012;21:587-96. http://dx.doi.org/10.1111/j.1365-294X.2011. 05376.x
45. Cellegari-Jacques SM, Grattapaglia D, Salzano FM, Salamoni SP, Crossetti SG, Ferreira ME, et al. Historical genetics: Spatiotemporal analysis of the formation of the Brazilian population. Am J Hum Biol. 2003;15:824-34. http://dx.doi.org/10.1002/ajhb.10217

46. Nazarenko SA, Puzyrev VP. Genetic drift of marker $Y$ chromosome del(Y)(q12) in Khanty from the lower Ob river. Hum Genet. 1985;71:100-2. http://dx.doi.org/10.1007/ BF00283361

47. Peltonen L, Jalanko A, Varilo T. Molecular genetics of the Finnish disease heritage. Hum Mol Genet. 1999;8:1913-23. http://dx.doi.org/10.1093/hmg/8.10.1913

48. Behar DM, Metspalu E, Kivisild T, Achilli A, Hadid Y, Tzur S, et al. The matrilineal ancestry of Ashkenazi Jewry: Portrait of a recent founder event. Am J Hum Genet. 2006;78:48797. http://dx.doi.org/10.1086/500307

49. Karafet TM, Méndez FL, Meilerman MB, Underhill PA, Zegura SL, Hammer MF. New binary polymorphisms reshape and increase resolution of the human Y chromosomal haplogroup tree. Genome Res. 2008;18:830-8. http://dx.doi. org/10.1101/gr.7172008

50. Balter M. Was North Africa the launch pad for modern human migrations? Science. 2011;331:20-3. http://dx.doi. org/10.1126/science.331.6013.20.

51. Excoffier L. Evolution of human mitochondrial DNA: Evidence for departure from a pure neutral model of populations at equilibrium. J Mol Evol. 1990;30:125-39. http://dx.doi.org/10.1007/BF02099939

52. Cox MP, Hammer MF. A question of scale: Human migrations writ large and small. BMC Biol. 2010;8:98. http://dx.doi. org/10.1186/1741-7007-8-98.

53. Cox MP, Woerner AE, Wall JD, Hammer MF. Intergenic DNA sequences from the human $X$ chromosome reveal high rates of global gene flow. BMC Genet. 2008;9:76. http:// dx.doi.org/10.1186/1471-2156-9-76

54. Marquet S, Abel L, Hillaire D, Dessein H, Kalil J, Feingold $\mathrm{J}$, et al. Genetic localization of a locus controlling the intensity of infection by Schistosoma mansoni on chromosome 5q31-q33. Nat Genet. 1996;14:181-4. http://dx. doi.org/10.1038/ng1096-181

55. Muller-Myhsok B, Stelma FF, Guisse-Sow F, Muntau B, Thye T, Burchard GD, et al. Further evidence suggesting the presence of a locus, on human chromosome 5q31-q33, influencing the intensity of infection with Schistosoma mansoni. Am J Hum Genet. 1997;61:452-4. http://dx.doi. org/10.1016/S0002-9297(07)64073-7

56. Greenwood CM, Fujiwara TM, Boothroyd LJ, Miller MA, Frappier D, Fanning EA, et al. Linkage of tuberculosis to chromosome 2q35 loci, including NRAMP1, in a large aboriginal Canadian family. Am J Hum Genet. 2000;67:40516. http://dx.doi.org/10.1086/303012

57. Siddiqui MR, Meisner S, Tosh K, Balakrishnan K, Ghei S, Fisher SE, et al. A major susceptibility locus for leprosy in India maps to chromosome 10p13. Nat Genet. 2001;27:43941. http://dx.doi.org/10.1038/86958

58. Mira MT, Alcais A, van Thuc N, Thai VH, Huong NT, Ba NN, et al. Chromosome $6 q 25$ is linked to susceptibility to leprosy in a Vietnamese population. Nat Genet. 2003; 33:412-5. http://dx.doi.org/10.1038/ng1096 